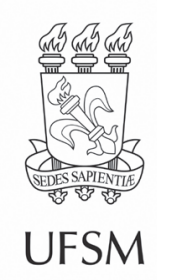

\title{
Artigos
}

\section{Potencial de regeneração natural de um plantio compensatório realizado em unidade de conservação urbana sob forte pressão antrópica}

\author{
Potential of natural regeneration of a compensatory planting carried out \\ in an urban conservation unit under strong anthropic pressure
}

\author{
Karina Cavalheiro Barbosa' ${ }^{\oplus}$ \\ Eduardo Luis Martins Catharino" $\odot$ \\ Luiz Mauro Barbosa" $\odot$ \\ Hilton Thadeu Zarate do Couto"II $\odot$ \\ Nelson Augusto dos Santos Junior" $\odot$ \\ 'Pesquisadora Autônoma, São Paulo, SP, Brasil \\ "Instituto de Botânica, São Paulo, SP, Brasil \\ '"'Universidade de São Paulo, Piracicaba, SP, Brasil
}

\section{RESUMO}

Áreas protegidas por lei representam uma das estratégias para conservação da biodiversidade, principalmente em regiões sob forte pressão antrópica. Porém, muitas dessas áreas protegidas apresentam barreiras impeditivas à regeneração natural e conhecê-las torna-se primordial. Nesse sentido, o objetivo deste trabalho foi verificar o potencial de regeneração de um plantio de restauração realizado em uma Unidade de Conservação, por meio da quantificação do aporte de diásporos e da formação de indivíduos jovens. Para isso, foram instalados coletores de sementes e parcelas de regeneração natural num plantio realizado no Parque Estadual das Fontes do Ipiranga, São Paulo, SP. Foram identificadas 54 espécies (41 arbóreas) e registrados 24.033 propágulos (17.775 da espécie invasora Urochloa decumbens) e quanto ao recrutamento de plântulas foram 58 espécies (16 arbóreas). O potencial de regeneração foi influenciado pelas condições da área, e a forte presença de gramíneas invasoras, aliada à baixa diversidade de espécies arbóreas identificadas na chuva de sementes e no recrutamento de plântulas, tem comprometido o processo.

Palavras-chave: Chuva de sementes; Gramíneas invasoras; Recrutamento de plântulas; Áreas protegidas 


\section{ABSTRACT}

Protected areas by the law represent one of the strategies for biodiversity conservation, especially in regions under strong human pressure. However, many of these protected areas present barriers that prevent natural regeneration and knowing them becomes essential. In this sense, the objective of this work was to verify the regeneration potential of a restoration plantation carried out in a Conservation Unit, by quantifying the contribution of diaspores and the formation of young individuals. Seed collectors and natural regeneration plots were launched in a plantation carried out at the Fontes do Ipiranga State Park, São Paulo, SP. 54 species (41 arboreal) were identified and 24,033 propagules $(17,775$ of the invasive species Urochloa decumbens) were registered and regarding seedling recruitment there were 58 species (16 arboreal). The regeneration potential was still greatly influenced by the conditions of the area and the strong presence of invasive grasses, combined with the low diversity of tree species identified in the seed rain and in the recruitment of seedlings, had compromised the process.

Keywords: Seed rain; Invasive grasses; Seedling recruitment; Protected areas

\section{INTRODUÇÃO}

As florestas tropicais são formações complexas, pela sua alta diversidade e rede de interações e dinâmica, porém isso as torna frágeis e vulneráveis, sujeitas a distúrbios de intensidade, duração e frequência variáveis (CHAZDON; URIARTE, 2016). Portanto, é necessária a proteção de florestas maduras remanescentes e fragmentos florestais, além da restauração florestal das áreas degradadas com o objetivo de manutenção ou ampliação da sua biodiversidade. Nesse sentido, a restauração florestal tem como objetivo aumentar a diversidade específica e superar as barreiras, como a competição com espécies invasoras e a baixa qualidade do solo, que impedem a regeneração natural após o plantio de mudas (HOOPER et al., 2005; BRANCALION et al., 2010; BARBOSA et al., 2017), assim como o restabelecimento de interações ecológicas complexas e as funções a elas associadas (SILVA et al., 2015).

A regeneração natural de florestas tropicais é, portanto, um processo extremamente importante para a sustentabilidade dos ecossistemas (LAMB et al., 2005; BRANCALION et al., 2015; CHAZDON e URIARTE, 2016) e essas barreiras impeditivas, como a ausência ou baixa disponibilidade de propágulos, falhas no recrutamento de plântulas, fatores de estresse, falhas no estabelecimento de interações, entre 
outros aspectos (ENGEL; PARROTA, 2003; BARBOSA; PIZO, 2006; RODRIGUES et al., 2011) representam um empecilho. Quando os obstáculos para a regeneração natural prevalecem, mesmo em áreas protegidas, a sucessão estagna e um novo tipo de ecossistema se desenvolve, geralmente dominado por gramíneas ou samambaias invasoras, prorrogando a recuperação da estrutura, da composição e dos serviços ecossistêmicos da floresta, tornando-se necessária a intervenção humana ativa, muitas vezes custosa, para que os processos sucessionais do ecossistema retornem (LAMB et al., 2005).

Assim, o estudo do potencial de regeneração em área de restauração florestal apresenta-se como uma importante ferramenta para o entendimento dos processos ligados à sucessão ecológica (FONSECA et al., 2017), particularmente em áreas protegidas e sob forte pressão antrópica. Diante desse cenário, Unidades de Conservação e outras áreas protegidas por lei representam, muitas vezes, a única estratégia para a conservação dos ecossistemas e das espécies que os constituem, a proteção de espécies ameaçadas, preservação de serviços ecossistêmicos ou ainda por razões culturais e sociais. O sucesso dessa estratégia de adoção de áreas protegidas é geralmente avaliado considerando apenas aspectos da flora, quanto à diversidade de espécies e à presença de espécies endêmicas e ameaçadas (RODRIGUES et al., 2004), pouco sendo considerados os demais seres vivos. No entanto, Geldmann et al. (2013) relatam sobre a falta de correlação entre o impacto das áreas protegidas sobre os habitats e espécies, sendo alarmante o pouco conhecimento quantitativo sobre o manejo e a melhora das condições da área protegida. Avaliar o aporte de propágulos, bem como o recrutamento inicial das plântulas, propiciaria a caracterização da dinâmica pela qual determinada Unidade de Conservação estaria passando.

Sendo assim, o presente trabalho teve como objetivo quantificar o aporte de diásporos e sua relação com a formação de indivíduos jovens, de forma correlacionálos com o processo de regeneração natural de um plantio realizado em uma Unidade de Conservação urbana. 


\section{MATERIAL E MÉTODO}

\section{1 Área de estudo}

A área de estudo está inserida no Parque Estadual das Fontes do Ipiranga (PEFI), situado entre os paralelos $23^{\circ} 38^{\prime} 08^{\prime \prime S}$ e $23^{\circ} 40^{\prime} 18^{\prime \prime S}$ e meridianos $46^{\circ} 36^{\prime} 48^{\prime \prime} \mathrm{W}$ e 46³8'00"S na região sudeste do Município de São Paulo (SP). É um dos maiores fragmentos florestais tropicais inseridos em área urbana, com 526 ha, protegendo nascentes e corpos d'água da bacia do Riacho do Ipiranga, porém sujeito a inúmeras perturbações como efeito de borda, incêndios, ilhas de calor, invasão biológica e poluição (SÃO PAULO, 2008). De acordo com os parâmetros que constam no Sistema Nacional de Unidades de Conservação (BRASIL, 2000), é uma unidade de proteção integral e, como tal, tem como objetivo principal a preservação de ecossistemas naturais de grande relevância ecológica, permitindo a realização de pesquisas científicas e o desenvolvimento de atividades de educação, recreação e turismo ecológico. O PEFI está inserido na zona do planalto paulista, de domínio do bioma de Mata Atlântica, pertencente à Floresta Ombrófila Densa e representantes da Floresta Estacional Semidecidual, com clima Cwb, segundo Koppen (BARROS et al., 2002; SANTOS; FUNARI, 2002; SÃO PAULO, 2008).

A área específica desta pesquisa refere-se a um plantio compensatório de seis hectares, em espaçamento $2 \mathrm{~m} \times 2 \mathrm{~m}$, realizado em 2015, no qual foram utilizadas 15.040 mudas, pertencentes a 98 espécies (46 pioneiras e 52 não pioneiras), plantio este que foi realizado em uma Zona de Uso Conflitante do PEFI. Seis meses após a execução do plantio, foram instalados os coletores de propágulos e as parcelas de recrutamento de plântulas para avaliar o potencial regenerativo da área.

\subsection{Método}

Para avaliar o aporte de propágulos, foram instalados 27 coletores de madeira, com 1,0m x1,0m, fundo com tela de sombreamento70\% (175 g.m $\mathrm{m}^{-2}$ ), suspensos 
$15 \mathrm{~cm}$ do solo e distribuídos de maneira sistematizada na área de seis hectares. A sistematização teve como objetivo verificar o efeito de borda do entorno sobre o plantio. Foram determinadas três áreas (relativas às três bordas do plantio) e, em cada uma, foram alocadas três linhas com três coletores em cada, de forma a totalizar os 27 coletores de chuva de sementes. As linhas distaram 5, 25 e 50 metros a contar da borda do plantio, sendo que os coletores distaram, no mínimo, 20 m entre si. Foi realizada coleta mensal do material depositado, por um período de dois anos.

No caso da avaliação do processo de regeneração natural, foram instaladas 27 parcelas, distribuídas nas mesmas faixas onde foram depositados os coletores de chuva de sementes, portanto distantes, também, pelo menos $20 \mathrm{~m}$ entre si. As parcelas, com dimensão de 1,0m x 1,0m, foram delimitadas com o uso de estacas e fitilho. A periodicidade da coleta foi semestral, por um período de dois anos e, em cada coleta, foram quantificadas e identificadas, no próprio local, todas as plântulas com no mínimo $10 \mathrm{~cm}$ de altura, presentes nas parcelas e, no caso das espécies ruderais que formavam touceiras, foi considerada a porcentagem de ocupação nas parcelas. A identificação das plantas foi realizada, quando possível, no momento de coleta dos dados, sem que as plantas fossem removidas, para que não houvesse interferência sobre o desenvolvimento deste nas parcelas. Aquelas plantas, cuja identificação não foi possível no local, foram buscados exemplares fora da parcela, herborizados e levados ao Núcleo de Pesquisa Curadoria do Herbário do Instituto de Botânica para consulta às exsicatas e aos especialistas.

\subsection{Análise dos dados}

O material depositado nos coletores de chuva de sementes foi quantificado e separado por morfo-espécies até a identificação final das espécies. Após identificação das sementes e frutos, os propágulos foram classificados de acordo com a origem (nativa do Brasil ou exótica), hábito de vida (árvore ou outras formas), classe sucessional (pioneira e não pioneira), guildas de dispersão (zoocórica, anemocórica e autocórica), 
baseadas em Van Der Pijl (1982), e quanto à origem das sementes (autóctone e alóctone), ou seja, se o propágulo veio de espécies com exemplares presentes ou de espécies sem ocorrência na área, respectivamente.

Os indivíduos regenerantes nas parcelas foram classificados quanto à origem (nativa ou exótica) e hábito de vida (árvore ou outras formas).

A análise dos dados referentes ao aporte de propágulos e ao recrutamento de plântulas foi semelhante. Foram obtidos os valores referentes à quantidade absoluta de propágulos e de plântulas, além da riqueza (número de espécies). Os valores mensais e semestrais obtidos para fins de verificação da época com maior aporte de diásporos e maior regeneração, respectivamente, foram comparados por meio da Análise de Variância (ANOVA) e posterior teste de médias, utilizando o software Sisvar (FERREIRA, 2011).

\section{RESULTADOS E DISCUSSÃO}

\subsection{Chuva de sementes}

Ao longo dos dois anos, foram registrados 24.033 propágulos $(890,11$ propágulosm ${ }^{-2}$ ou 37,08 propágulosm ${ }^{-2} \mathrm{mês}^{-1}$ ), dos quais 17.775 pertencem a uma única espécie invasora (Urochloa decumbens) e apenas 5.961 são arbóreas (220,77 propágulosm-2 ou 9,20 propágulosm-2mês ${ }^{-1}$ ). Foram identificadas 54 espécies (27 famílias), sendo 41 espécies arbóreas, 37 nativas (5.630 sementes) e 4 exóticas (331 sementes), sendo 32 pioneiras, 19 zoocóricas e 15 alóctones (Tabela 1). Dentre as 27 famílias identificadas na chuva de sementes, Fabaceae teve a maior relação de espécies identificadas (09) e outras famílias não tiveram representatividade superior a quatro espécies neste estudo. A família Poaceae teve a maior quantidade de sementes amostradas, sendo 17.775 pertencentes a uma única espécie Urochloa decumbens, seguida da Moraceae com 1.271 sementes pertencentes a apenas duas espécies de Ficus sp. e Anarcadiaceae com 1.260 sementes, das quais 1.251 referem-se à Schinus terebinthifolia. 
Tabela 1 - Quantidade de diásporos, ao longo dos meses e durante um ano, das espécies encontradas na chuva de sementes, classificados de acordo com o hábito de vida e origem

\begin{tabular}{|c|c|c|c|c|c|c|c|c|c|c|c|c|c|c|c|c|c|}
\hline \multirow{2}{*}{ Família/Espécies } & \multirow{2}{*}{$\mathbf{F}$} & \multirow{2}{*}{$\mathbf{s}$} & \multirow{2}{*}{ D } & \multirow{2}{*}{0} & \multicolumn{12}{|c|}{ Meses } & \multirow{2}{*}{ Total } \\
\hline & & & & & s & 0 & $\mathbf{N}$ & D & J & $\mathbf{F}$ & M & A & $\mathbf{M}$ & J & $J$ & A & \\
\hline \multicolumn{18}{|l|}{ Anacardiaceae } \\
\hline Schinus terebinthifolia Raddi & $\mathrm{N}$ & $\mathrm{P}$ & Z & $\mathrm{AU}$ & 3 & 0 & 0 & 506 & 515 & 71 & 12 & 19 & 51 & 26 & 12 & 36 & 1251 \\
\hline Lithraea molleoides (Vell.) Engl. & $\mathrm{N}$ & $\mathrm{P}$ & z & $\mathrm{AU}$ & 0 & 0 & 0 & 0 & 0 & 0 & 0 & 0 & 0 & 0 & 9 & 0 & 9 \\
\hline \multicolumn{18}{|l|}{ Annonaceae } \\
\hline Duguetia lanceolata A.St.-Hil. & $\mathrm{N}$ & $\mathrm{N}$ & Z & $\mathrm{AL}$ & 0 & 7 & 2 & 62 & 1 & 0 & 0 & 1 & 0 & 0 & 1 & 0 & 74 \\
\hline Annona sylvatica A.St.-Hil. & N & $\mathrm{P}$ & z & $\mathrm{AL}$ & 0 & 1 & 5 & 4 & 7 & 0 & 0 & 0 & 0 & 0 & 0 & 0 & 17 \\
\hline \multicolumn{18}{|l|}{ Arecaceae } \\
\hline \multicolumn{18}{|l|}{ Archontophoenix } \\
\hline cunninghamiana & $E$ & $P$ & Z & $\mathrm{AU}$ & 2 & 2 & 0 & 5 & 29 & 14 & 7 & 2 & 1 & 4 & 2 & 1 & 69 \\
\hline \multicolumn{18}{|l|}{ (H. Wendl.) H. Wendl. \& Drude. } \\
\hline \multicolumn{18}{|l|}{ Asteraceae } \\
\hline \multicolumn{18}{|l|}{ Vernonia polyanthes (Spreng.) } \\
\hline \multicolumn{18}{|l|}{ Less. } \\
\hline \multicolumn{18}{|l|}{ Vernonanthura discolor } \\
\hline (Spreng.) H. Rob. & $N$ & $\mathrm{P}$ & A & $\mathrm{AL}$ & 0 & 0 & 0 & 0 & 3 & 0 & 56 & 655 & 53 & 14 & 5 & 1 & 787 \\
\hline Taraxacum officinale F.H. Wigg. & EO & - & - & - & 0 & 0 & 0 & 0 & 0 & 0 & 0 & 52 & 0 & 0 & 0 & 0 & 52 \\
\hline \multicolumn{18}{|l|}{ Bignoniaceae } \\
\hline $\begin{array}{l}\text { Handroanthus heptaphyllus } \\
\text { (Vell.) Mattos }\end{array}$ & $\mathrm{N}$ & $\mathrm{N}$ & A & $\mathrm{AU}$ & 0 & 3 & 1 & 0 & 0 & 0 & 0 & 0 & 0 & 0 & 0 & 0 & 4 \\
\hline \multicolumn{18}{|l|}{ Amphilophium crucigerum } \\
\hline (L.) L. G. Lohmann & $\mathrm{O}$ & - & - & - & 6 & 2 & 1 & 0 & 0 & 0 & 0 & 0 & 0 & 0 & 0 & 1 & 10 \\
\hline Handroanthus sp & $\mathrm{N}$ & $\mathrm{N}$ & A & $\mathrm{AU}$ & 0 & 0 & 0 & 0 & 1 & 0 & 0 & 0 & 0 & 0 & 0 & 0 & 1 \\
\hline \multicolumn{18}{|l|}{ Bixaceae } \\
\hline \multicolumn{18}{|l|}{ Bixa orellana var. leiocarpa } \\
\hline (Kuntze) Standl. \& L. O. & $\mathrm{N}$ & $\mathrm{P}$ & Z & $\mathrm{AL}$ & 0 & 0 & 0 & 0 & 0 & 0 & 0 & 0 & 0 & 0 & 0 & 159 & 159 \\
\hline Williams & & & & & & & & & & & & & & & & & \\
\hline Clethraceae & & & & & & & & & & & & & & & & & \\
\hline Clethra scabra Pers. & $N$ & $P$ & A & $\mathrm{AL}$ & 0 & 0 & 0 & 0 & 0 & 101 & 0 & 0 & 0 & 0 & 0 & 0 & 101 \\
\hline Cluseaceae & & & & & & & & & & & & & & & & & \\
\hline $\begin{array}{l}\text { Garcinia gardneriana } \\
\text { (Planch. \&Triana) Zappi }\end{array}$ & $\mathrm{N}$ & $\mathrm{N}$ & Z & $\mathrm{AL}$ & 0 & 0 & 1 & 1 & 0 & 2 & 0 & 1 & 0 & 0 & 0 & 0 & 5 \\
\hline Curcubitaceae & & & & & & & & & & & & & & & & & \\
\hline Cayaponia sp & $\mathrm{O}$ & - & - & - & 0 & 0 & 15 & 0 & 0 & 0 & 0 & 0 & 0 & 0 & 0 & 0 & 15 \\
\hline Euphorbiaceae & & & & & & & & & & & & & & & & & \\
\hline Croton urucurana Baill. & $N$ & $P$ & $\mathrm{Au}$ & $\mathrm{AU}$ & 3 & 1 & 0 & 1 & 0 & 0 & 4 & 2 & 0 & 11 & 1 & 0 & 23 \\
\hline Croton floribundus Spreng. & $\mathrm{N}$ & $\mathrm{P}$ & $\mathrm{Au}$ & $A U$ & 0 & 0 & 0 & 18 & 2 & 0 & 0 & 0 & 0 & 0 & 0 & 0 & 20 \\
\hline Sebastiania commersoniana & & & & & & & & & & & & & & & & & \\
\hline (Baill.) L.B.Sm. \& Downs & $N$ & $\mathrm{P}$ & $\mathrm{Au}$ & $\mathrm{AL}$ & 0 & 0 & 0 & 0 & 0 & 0 & 0 & 0 & 0 & 1 & 6 & 0 & 7 \\
\hline
\end{tabular}


Tabela 1 - Continuação

\begin{tabular}{|c|c|c|c|c|c|c|c|c|c|c|c|c|c|c|c|c|c|}
\hline \multirow{2}{*}{ Família/Espécies } & \multirow{2}{*}{$\mathbf{F}$} & \multirow{2}{*}{ s } & \multirow{2}{*}{ D } & \multirow{2}{*}{0} & \multicolumn{12}{|c|}{ Meses } & \multirow{2}{*}{ Total } \\
\hline & & & & & $\mathrm{s}$ & 0 & $N$ & D & $\mathrm{J}$ & $F$ & $M$ & A & $M$ & J & $\mathrm{J}$ & A & \\
\hline \multicolumn{18}{|l|}{ Fabaceae } \\
\hline $\begin{array}{l}\text { Peltophorum dubium } \\
\text { (Spreng.) Taub. }\end{array}$ & $N$ & $P$ & $\mathrm{Au}$ & $\mathrm{AU}$ & 33 & 28 & 2 & 5 & 1 & 1 & 3 & 1 & 22 & 4 & 5 & 1 & 106 \\
\hline Senna macranthera (DC. ex & & & & & & & & & & & & & & & & & \\
\hline (ollad.) & $N$ & $P$ & $\mathrm{Au}$ & $\mathrm{AU}$ & 0 & 0 & 0 & 0 & 0 & 0 & 2 & 0 & 0 & 0 & 0 & 0 & 2 \\
\hline \multicolumn{18}{|l|}{ H. S. Irwin \& Barneby } \\
\hline $\begin{array}{l}\text { Cassia ferruginea (Schrad.) } \\
\text { Schrad. ex DC. }\end{array}$ & N & N & $\mathrm{Au}$ & $\mathrm{AU}$ & 24 & 1 & 0 & 0 & 0 & 0 & 0 & 0 & 0 & 1 & 11 & 51 & 88 \\
\hline Mimosa scabrella Benth. & $\mathrm{N}$ & $P$ & $\mathrm{Au}$ & $\mathrm{AU}$ & 41 & 96 & 14 & 4 & 1 & 0 & 0 & 0 & 0 & 0 & 7 & 174 & 337 \\
\hline Mimosa sp & - & - & - & - & 2 & 4 & 3 & 2 & 1 & 0 & 1 & 0 & 1 & 0 & 0 & 6 & 20 \\
\hline $\begin{array}{l}\text { Piptadenia gonoacantha (Mart.) } \\
\text { J. F. Macbr. }\end{array}$ & $\mathrm{N}$ & $P$ & $\mathrm{Au}$ & AU & 2 & 1 & 0 & 1 & 0 & 0 & 0 & 0 & 0 & 0 & 0 & 1 & 5 \\
\hline $\begin{array}{l}\text { Mimosa bimucronata (DC.) } \\
\text { Kuntze }\end{array}$ & $\mathrm{N}$ & $P$ & $\mathrm{Au}$ & $\mathrm{AU}$ & 0 & 9 & 1 & 0 & 0 & 0 & 0 & 0 & 0 & 0 & 0 & 0 & 10 \\
\hline Inga sp & N & $P$ & z & AU & 0 & 2 & 6 & 13 & 0 & 0 & 0 & 0 & 0 & 0 & 0 & 0 & 21 \\
\hline Mimosa caesalpiniifolia Benth. & N & $P$ & A & AL & 0 & 0 & 0 & 0 & 0 & 0 & 0 & 0 & 80 & 127 & 43 & 72 & 322 \\
\hline Lauraceae & & & & & & & & & & & & & & & & & \\
\hline Lauraceae 01 & - & - & - & - & 0 & 0 & 0 & 0 & 0 & 0 & 0 & 0 & 0 & 0 & 5 & 0 & 5 \\
\hline Malvaceae & & & & & & & & & & & & & & & & & \\
\hline $\begin{array}{l}\text { Ochroma pyramidale } \\
\text { (Cav. ex Lam.) Urb. }\end{array}$ & N & $P$ & A & AL & 10 & 1 & 0 & 4 & 0 & 0 & 0 & 0 & 0 & 0 & 0 & 0 & 15 \\
\hline Heliocarpus popayanensis Kunth & $\mathrm{N}$ & $P$ & A & $A U$ & 1 & 7 & 3 & 0 & 0 & 0 & 0 & 0 & 0 & 0 & 0 & 0 & 11 \\
\hline $\begin{array}{l}\text { Ceiba speciosa (A. St.-Hil.) } \\
\text { Ravenna }\end{array}$ & $\mathrm{N}$ & $\mathrm{N}$ & A & $\mathrm{AU}$ & 0 & 0 & 0 & 2 & 0 & 5 & 0 & 0 & 1 & 0 & 9 & 13 & 30 \\
\hline Melastomaceae & & & & & & & & & & & & & & & & & \\
\hline $\begin{array}{l}\text { Tibouchina granulosa (Desr.) } \\
\text { Cogn. }\end{array}$ & N & $P$ & A & AL & 8 & 4 & 6 & 7 & 4 & 0 & 48 & 15 & 6 & 4 & 7 & 1 & 110 \\
\hline Meliaceae & & & & & & & & & & & & & & & & & \\
\hline Melia azedarach L. & $\mathrm{E}$ & $P$ & z & AU & 1 & 0 & 0 & 0 & 0 & 0 & 2 & 0 & 1 & 6 & 1 & 0 & 11 \\
\hline Moraceae & & & & & & & & & & & & & & & & & \\
\hline Ficus sp 1 & N & $P$ & z & $\mathrm{AU}$ & 131 & 8 & 82 & 174 & 75 & 12 & 97 & 21 & 3 & 0 & 49 & 51 & 703 \\
\hline Ficus sp 2 & $N$ & $\mathrm{P}$ & $\mathrm{z}$ & $A U$ & 60 & 79 & 3 & 214 & 108 & 64 & 5 & 13 & 0 & 1 & 7 & 14 & 568 \\
\hline Myrtaceae & & & & & & & & & & & & & & & & & \\
\hline Psidium sp & $N$ & $P$ & z & $\mathrm{AL}$ & 3 & 0 & 2 & 0 & 0 & 0 & 0 & 4 & 11 & 3 & 11 & 0 & 34 \\
\hline Eugenia sp & $N$ & $N$ & z & $A U$ & 0 & 0 & 0 & 17 & 0 & 0 & 0 & 0 & 0 & 0 & 0 & 0 & 17 \\
\hline Myrtaceae 01 & - & - & - & - & 0 & 2 & 0 & 0 & 0 & 0 & 0 & 0 & 0 & 0 & 0 & 0 & 2 \\
\hline Myrtaceae 02 & - & - & - & - & 0 & 0 & 0 & 4 & 0 & 0 & 0 & 0 & 0 & 0 & 0 & 0 & 4 \\
\hline Phyllanthaceae & & & & & & & & & & & & & & & & & \\
\hline $\begin{array}{l}\text { Hyeronima alchorneoides } \\
\text { Allemão }\end{array}$ & $N$ & $\mathrm{P}$ & z & AL & 21 & 0 & 0 & 0 & 0 & 60 & 77 & 4 & 12 & 39 & 53 & 22 & 288 \\
\hline Pinaceae & & & & & & & & & & & & & & & & & \\
\hline Pinus sp & $\mathrm{E}$ & $P$ & A & $A U$ & 9 & 0 & 0 & 0 & 0 & 0 & 0 & 0 & 0 & 0 & 0 & 32 & 41 \\
\hline Pinus sylvestris L. & $\mathrm{E}$ & $P$ & A & $\mathrm{AU}$ & 11 & 0 & 0 & 4 & 1 & 0 & 1 & 72 & 80 & 21 & 4 & 16 & 210 \\
\hline
\end{tabular}


Tabela 1 - Conclusão

\begin{tabular}{|c|c|c|c|c|c|c|c|c|c|c|c|c|c|c|c|c|c|}
\hline \multirow{2}{*}{ Família/Espécies } & \multirow{2}{*}{$\mathbf{F}$} & \multirow{2}{*}{$s$} & \multirow{2}{*}{ D } & \multirow{2}{*}{0} & \multicolumn{12}{|c|}{ Meses } & \multirow{2}{*}{ Total } \\
\hline & & & & & $s$ & 0 & $N$ & D & J & $\mathbf{F}$ & M & A & $M$ & J & $\mathrm{J}$ & A & \\
\hline \multicolumn{18}{|l|}{ Poaceae } \\
\hline $\begin{array}{l}\text { Urochloa decumbens } \\
\text { (Stapf) R.D. Webster }\end{array}$ & 0 & - & - & - & 475 & 1804 & 1625 & 1222 & 868 & 2019 & 2450 & 1308 & 1676 & 1364 & 1354 & 1587 & 17752 \\
\hline \multicolumn{18}{|l|}{ Primulaceae } \\
\hline Myrsine gardneriana A.DC. & N & $\mathrm{P}$ & $\mathrm{z}$ & $\mathrm{AU}$ & 0 & 3 & 0 & 61 & 40 & 5 & 1 & 0 & 0 & 0 & 0 & 0 & 110 \\
\hline \multicolumn{18}{|l|}{ Rubiaceae } \\
\hline Psychotria viridis Ruiz \& Pav. & $\mathrm{N}$ & $\mathrm{P}$ & A & AL & 0 & 0 & 0 & 0 & 0 & 0 & 0 & 0 & 0 & 0 & 41 & 16 & 57 \\
\hline \multicolumn{18}{|l|}{ Santalaceae } \\
\hline Phoradendron sp & 0 & - & - & - & 55 & 10 & 1 & 0 & 0 & 0 & 0 & 0 & 0 & 1 & 4 & 18 & 89 \\
\hline \multicolumn{18}{|l|}{ Sapindaceae } \\
\hline Sapindus saponaria L. & $\mathrm{N}$ & $\mathrm{N}$ & $\mathrm{z}$ & AL & 0 & 1 & 0 & 0 & 0 & 0 & 0 & 0 & 0 & 0 & 0 & 0 & 1 \\
\hline $\begin{array}{l}\text { Allophylus edulis (A. St.-Hill. et } \\
\text { al.) Hieron. ex Niederl. }\end{array}$ & $N$ & $P$ & z & $\mathrm{AU}$ & 0 & 1 & 0 & 0 & 0 & 0 & 0 & 0 & 0 & 0 & 0 & 0 & 1 \\
\hline \multicolumn{18}{|l|}{ Solanaceae } \\
\hline Solanum americanum Mill. & 0 & - & - & - & 0 & 1 & 1 & 32 & 41 & 0 & 0 & 9 & 0 & 0 & 0 & 0 & 84 \\
\hline \multicolumn{18}{|l|}{ Symplocaceae } \\
\hline Symplocos sp & N & N & $\mathrm{z}$ & AL & 0 & 1 & 0 & 1 & 1 & 2 & 0 & 0 & 0 & 0 & 0 & 0 & 5 \\
\hline \multicolumn{18}{|l|}{ Verbenaceae } \\
\hline $\begin{array}{l}\text { Citharexylum myrianthum } \\
\text { Cham. }\end{array}$ & $N$ & $P$ & $\mathrm{z}$ & $\mathrm{AU}$ & 0 & 0 & 0 & 0 & 16 & 10 & 15 & 1 & 0 & 1 & 0 & 0 & 43 \\
\hline \multicolumn{18}{|l|}{ Não identificadas } \\
\hline Morfoespécie 01 & - & - & - & - & 0 & 0 & 1 & 0 & 0 & 0 & 0 & 0 & 26 & 0 & 0 & 0 & 27 \\
\hline Morfoespécie 02 & - & - & - & - & 1 & 0 & 0 & 0 & 1 & 0 & 1 & 0 & 0 & 0 & 0 & 1 & 4 \\
\hline Morfoespécie 03 & - & - & - & - & 0 & 0 & 0 & 0 & 0 & 0 & 0 & 0 & 0 & 0 & 8 & 0 & 8 \\
\hline Total sementes & & & & & 1038 & 2228 & 1778 & 2364 & 1716 & 2366 & 2782 & 2180 & 2024 & 1628 & 1655 & 2274 & 24033 \\
\hline Total espécies & & & & & 23 & 27 & 21 & 25 & 20 & 13 & 18 & 18 & 14 & 17 & 23 & 22 & 54 \\
\hline $\begin{array}{l}\text { Total sementes (excluindo } \\
\text { Urochloa decumbens) }\end{array}$ & & & & & 563 & 424 & 153 & 1142 & 848 & 347 & 332 & 872 & 348 & 264 & 301 & 687 & 6281 \\
\hline $\begin{array}{l}\text { Total espécies (excluindo } \\
\text { Urochloa decumbens) }\end{array}$ & & & & & 22 & 26 & 20 & 24 & 19 & 12 & 17 & 17 & 13 & 16 & 22 & 21 & 53 \\
\hline
\end{tabular}

Fonte: Autores (2020)

Em que: $(\mathrm{F}): \mathrm{N}$ = árvore nativa, $\mathrm{E}$ = árvore exótica e $\mathrm{EO}=$ outra exótica; grupo sucessional $(\mathrm{S}): \mathrm{P}=$ pioneira e NP = não pioneira; síndrome de dispersão (D): $A$ = anemocoria, $A u$ = autocoria, $Z$ = zoocoria; e origem do propágulo $(\mathrm{O}): \mathrm{AU}=$ autóctone, $\mathrm{AL}=$ alóctone.

Apesar do domínio quantitativo das sementes da gramínea invasora Urochloa decumbens na chuva de sementes, houve mais espécies nativas exóticas identificadas 
e ainda um crescente aporte de sementes na área, provavelmente devido ao remanescente florestal adjacente, ao início do processo de formação estrutural no local, oferecendo mudanças importantes quanto aos recursos de composição e estrutura de vegetação e oferta de abrigo e alimento à fauna. Isso amplia a quantidade de recursos de alimentos, bem como a estrutura da vegetação pela disponibilidade de poleiros e abrigo, atraindo um número maior de animais frugívoros com maior frequência à área plantada (CHAZDON; URIARTE, 2016).

$\mathrm{Na}$ fase inicial de colonização e formação de estrutura da vegetação, na composição da chuva de sementes foram identificadas espécies pioneiras dominantes e autóctones, um padrão também observado por Barbosa e Pizo (2006), Melo et al. (2006) e Battilani (2010). Os maiores registros com relação às espécies arbóreas nativas, tanto para o número de sementes quanto para o número de espécies, foram referentes à síndrome de dispersão zoocórica, um padrão típico para florestais tropicais (HOWE; SMALLWOOD, 1982; CÔRTES; URIARTE, 2013).

Com relação às famílias identificadas, a mais rica devido ao número de espécies identificadas foi Fabaceae uma das mais representativas em espécies nas florestas estacionais semidecíduas sob domínio de Mata Atlântica nas regiões sul, sudeste e centro-oeste do Brasil (LEITÃO-FILHO, 1987; OLIVEIRA-FILHO; FONTES, 2000). Poaceae foi a família que teve o maior número de sementes registradas no período, $74 \%$ da amostragem total da chuva de sementes, pertencentes à única espécie registrada (Urochloa decumbens) evidenciando que a área ainda apresenta condições favoráveis à invasão dessa espécie, o que provavelmente estava reduzindo a regeneração natural na área. A agressividade do gênero em plantios de restauração foi relatada por Santos et al. (2020).

Analisando a quantidade de propágulos e sua distribuição ao longo do período estudado (Figura 1), o número coletado por mês variou de 499 (18,48 propágulosm²) a 1.795 (66,48 propágulosm²) ou 0 a 393 (14,55 propágulosm²) quando excluídas as sementes de Urochloa decumbens, sendo notória a interferência dessa espécie na 
área. Os maiores registros de propágulos no período foram nos meses de março, abril e dezembro de 2016, com a presença marcante das sementes de Urochloa decumbens, principalmente em abril do ano em questão. Excluindo o número de sementes de Urochloa decumbens, os maiores registros foram nos meses de dezembro de 2015, devido as sementes de Schinus terebinthifolia (393), Ficus sp. (215) e Duguetia lanceolata (61), e abril de 2016, devido às sementes de Vernonia discolor (655) e Pinus sylvestris (70), porém no mesmo período (dezembro e abril) do ano subsequente, o número de sementes amostradas (excluindo a Urochloa decumbens) foi muito inferior (menos que a metade). Não houve efeito da distância dos coletores de chuva de sementes e das parcelas de regeneração a partir da borda do plantio, razão pela qual foram avaliados conjuntamente.

Figura 1 - Número de sementes amostradas mensalmente durante dois anos, incluindo e excluindo Urochloa decumbens, nos coletores distribuídos ao longo da área de plantio no PEFI (SP). Valores médios seguidos pela mesma letra não diferem entre si em nível de $5 \%$ pelo teste de Scott-Knott

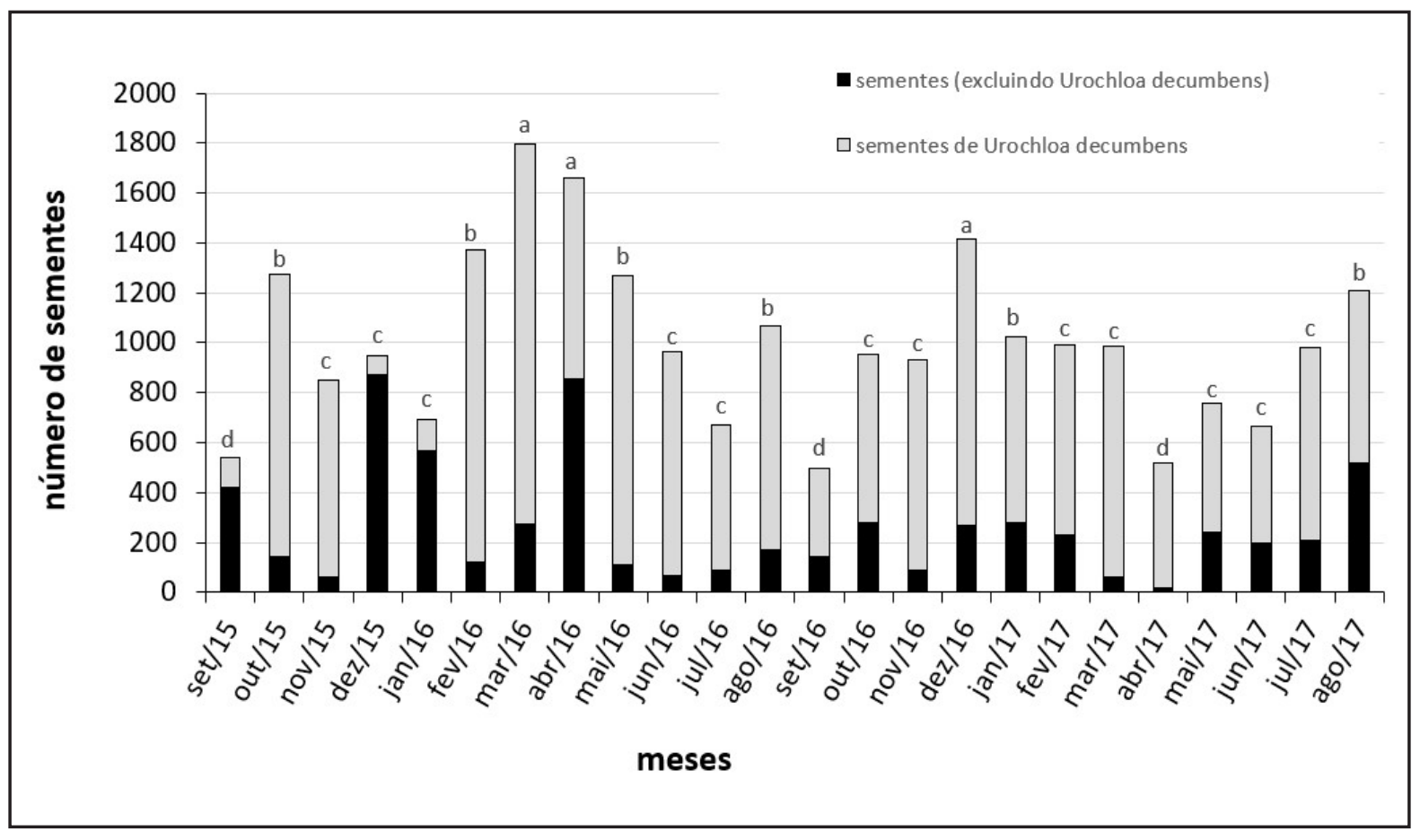

Fontes: Autores (2020) 
Já com relação à quantidade e distribuição das espécies ao longo do período estudado (Figura 2), o número variou de sete (0,26 espéciesm²) a 22 (2,45 espéciesm²), sendo que Urochloa decumbens foi registrada em todas as coletas. Os meses que tiveram os maiores registros de espécies amostradas no período foram outubro de 2016 e julho de 2017, respectivamente 22 e 21 espécies. Em outubro de 2016, foi registrado o dobro de espécies (22) quando comparado com o registro de espécies em outubro de 2015 (11 espécies) e julho de 2017 tendo o registro de 21 espécies. Também se observa quase o dobro no mesmo mês do ano anterior (13 espécies).

Figura 2 - Número de espécies amostradas mensalmente, e durante dois anos, nos coletores distribuídos ao longo da área de plantio no PEFI (SP). Valores médios seguidos pela mesma letra não diferem entre si em nível de 5\% pelo teste de Scott-Knott

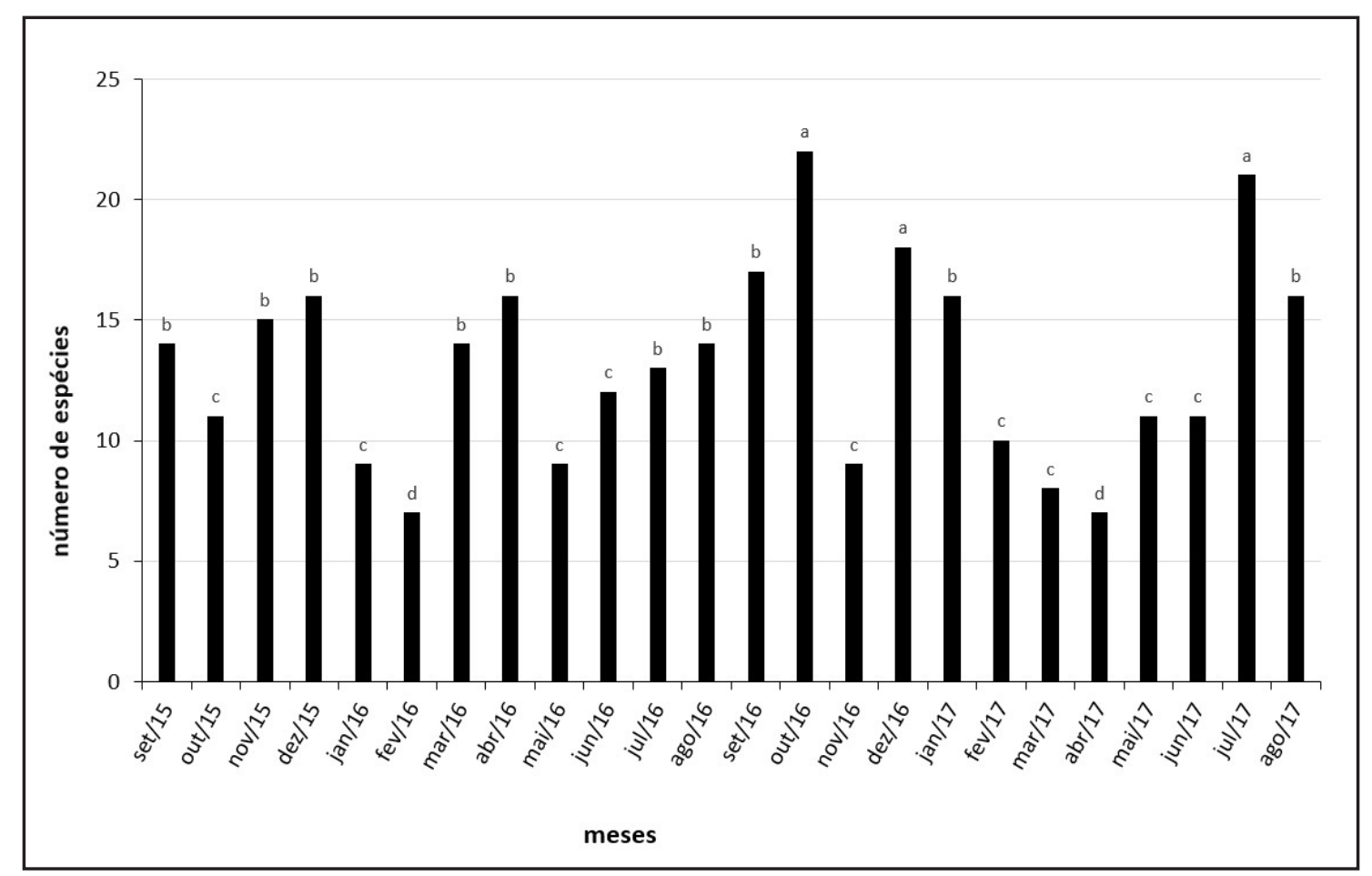

Fonte: Autores (2020)

Os maiores registros de propágulos (excluindo as sementes de Urochloa decumbens) ocorreram em épocas chuvosas e de elevadas temperaturas, o que ocorre no final da primavera estendendo-se até o final do verão na região onde o 
PEFI está inserido (SANTOS; FUNARI, 2002). Em dezembro de 2015, o número elevado de sementes ocorreu devido à Schinus terebinthifolia e Ficus sp., que frutificaram no período, atraindo a fauna dispersora de sementes e aumentando a possibilidade de outras espécies (como a D. lanceolata) chegarem a área plantada. Em abril de 2016, 0 número de sementes ocorreu devido à dispersão de espécies anemocóricas (Vernonia discolor e Pinus sylvestris) favorecida pela seca desse período. O conhecimento da variação do aporte de sementes, ao longo de um período determinado e superior a um ano, é fundamental para a compreensão dos processos reprodutivos e de dinâmica da vegetação, fornecendo informações importantes sobre abundância e riqueza (GROMBONE-GUARATINI; RODRIGUES, 2002), aumentando as chances de sementes de espécies com baixa densidade reprodutiva ou de limitada dispersão de sementes de serem amostradas (BARBOSA; PIZO, 2006). De acordo com Battilani (2010) e Silva et al. (2018), as variações no aporte de sementes e espécies zoocóricas de florestas tropicais estão associadas a fatores ambientais, como umidade, temperatura e severidade da sazonalidade climática.

A restauração florestal sofre influências da paisagem onde está inserida. De acordo com Leite et al. (2013), em levantamento realizado em trabalhos de restauração em diversas paisagens no mundo, $84 \%$ dos estudos demonstram que elas podem exercer uma influência positiva sobre a eficácia da restauração. Contudo, neste caso, fica evidente a vulnerabilidade da área em processo de restauração em decorrência da expressiva quantidade de sementes da gramínea exótica invasora (Urochloa decumbens) presente na área plantada.

\subsection{Recrutamento de plântulas}

Ao longo dos dois anos foram registrados cerca de 70 indivíduos regenerantes por semestre, o correspondente a 2,61 plântulasm². No primeiro ano observou-se uma crescente ocupação da área relacionada à quantidade de indivíduos, 52 plântulas registradas em fevereiro de 2016 e 121 plântulas em agosto de 2016, assim como 
aumentou a riqueza de espécies identificadas, respectivamente 30 e 36 espécies. Depois disso, em fevereiro e agosto de 2017, o número de plântulas e de espécies identificadas nas parcelas diminuiu, respectivamente, 60 indivíduos e 21 espécies e 49 indivíduos e 20 espécies (Tabela 2).Notou-se que entre o primeiro e o último registro de plântulas houve redução em 50\% de plântulas pertencentes a outros hábitos de vida que não a arbórea e considerável aumento (em 100\%) do registro e ocupação por espécies arbóreas. Foram identificadas, no período, 58 espécies (18 famílias), das quais apenas 16 são arbóreas, sendo quatro autóctones a área (Acnistus arborescens, Erythrina crista-galli, Eugenia uniflora e Vernonia phosphorica) e outras três também identificadas na chuva de sementes (Mimosa sp., Solanum americanum e Vernonia sp.). Dentre as famílias com maior representatividade destacaram-se Asteraceae, com 21 espécies, Fabaceae, com oito espécies, e Poaceae, com seis espécies.

Tabela 2 - Quantidade de plântulas, coletadas a cada seis meses, das espécies encontradas na regeneração natural, classificadas de acordo com a hábito de vida (H) e a origem $(\mathrm{O})$

\begin{tabular}{|c|c|c|c|c|c|c|}
\hline & \multirow[b]{2}{*}{$\mathbf{H}$} & \multirow[b]{2}{*}{0} & \multicolumn{4}{|c|}{ Semestre } \\
\hline & & & Fev/16 & Ago/16 & Fev/17 & Ago/17 \\
\hline \multicolumn{7}{|l|}{ Araliaceae } \\
\hline Hydrocotyle sp & 0 & $\mathrm{~N}$ & & 5 & & \\
\hline \multicolumn{7}{|l|}{ Asteraceae } \\
\hline Achyrocline satureioides (Lam.) DC. & 0 & $\mathrm{~N}$ & & 1 & 3 & 1 \\
\hline Artemisia cf. verlotorum Lamotte & O & $\mathrm{N}$ & & 1 & & \\
\hline Baccharis dracunculifolia DC. & $\mathrm{T}$ & $\mathrm{N}$ & 3 & 8 & 7 & 5 \\
\hline Bidens alba (L.) DC. & $\mathrm{O}$ & $\mathrm{N}$ & 1 & & & 1 \\
\hline Bidens pilosa $\mathrm{L}$. & $\mathrm{O}$ & $\mathrm{N}$ & 1 & 4 & 8 & 4 \\
\hline Cyrtocymura cf scorpioides (Lam.) H.Rob. & $\mathrm{T}$ & $\mathrm{N}$ & & & & 1 \\
\hline Eclipta alba $\mathrm{L}$. & O & $\mathrm{N}$ & 5 & & & \\
\hline Elephantopus mollis Kunth. & 0 & $\mathrm{~N}$ & & 10 & & \\
\hline Emilia sonchifolia (L.) DC. ExWight & O & $\mathrm{N}$ & 1 & 20 & 1 & 4 \\
\hline Galinsoga cf quadriradiata Ruiz \&Pav. & 0 & $\mathrm{~N}$ & 1 & & & \\
\hline Gamochaeta americana (Mill.) Wedd. & $\mathrm{O}$ & $\mathrm{N}$ & 1 & & & \\
\hline Gochnatia polymorpha (Less.) Cabr. & $T$ & $\mathrm{~N}$ & & & 2 & 2 \\
\hline Mikania cf. cordifolia(L.f.) Willd. & $\mathrm{O}$ & $\mathrm{N}$ & 1 & & 2 & \\
\hline
\end{tabular}


Tabela 2 - Continuação

Semestre

\begin{tabular}{llllll} 
H & 0 & Fev/16 & Ago/16 & Fev/17 & Ago/17 \\
\cline { 2 - 5 }
\end{tabular}

Araliaceae

Parthenium hysterophorus L.

Senecio brasiliensis Less.

\begin{tabular}{|c|c|c|c|c|c|}
\hline 0 & $\mathrm{~N}$ & & 1 & & \\
\hline O & $\mathrm{N}$ & & 5 & & \\
\hline 0 & $\mathrm{~N}$ & & 3 & 1 & 1 \\
\hline $\mathrm{T}$ & $\mathrm{N}$ & & & & \\
\hline $\mathrm{T}$ & $\mathrm{N}$ & 2 & 1 & 10 & 6 \\
\hline $\mathrm{T}$ & $\mathrm{N}$ & 1 & 2 & & \\
\hline $\mathrm{T}$ & $\mathrm{N}$ & 3 & 6 & 5 & 9 \\
\hline 0 & $\mathrm{E}$ & & 13 & 3 & 4 \\
\hline
\end{tabular}

Youngia japonica (L.) DC.

$\begin{array}{llll}\mathrm{O} & \mathrm{N} & 2 & 2\end{array}$

Blechnum serrulatum Rich.

Cyperaceae

Cyperus sp

Rhynchospora corymbosa (L.) Britton

$\begin{array}{llll}\mathrm{O} & \mathrm{N} & 5 & 1\end{array}$

Euphorbiaceae

Alchornea sidifolia Müll. Arg.

$\begin{array}{llll}\mathrm{O} & \mathrm{N} & 6 & 2\end{array}$

Croton lundianus (Didr.) Müll. Arg.

Euphorbia parviflora L.

$\mathrm{T}-\mathrm{N}$

$\mathrm{O} \quad \mathrm{N}$

O E

Ricinus communis L.

O $E \quad 1$

Fabaceae

Chamaecrista rotundifolia (Pers.) Greene

O $\quad E \quad 1$

Crotalaria sp

Desmodium cf adscendens (Sw.) DC.

$\mathrm{O} N$

* Erythrina crista-galli L.

$\mathrm{O} N$

T N

Indigofera cf. suffruticosa Mill.

O N

$\mathrm{T} \quad \mathrm{N}$

** Mimosa sp

O E

Neonotonia wightii (Wight \& Arn.) Lackey

O N

Iridaceae

Sisyrinchium sp

O N

Lamiaceae

Hyptis sp

O N

Lythraceae

Cupheacfmesostemon (Koehne) L.

$\mathrm{O} N$

2

Continua ... 
Tabela 2 - Conclusão

\begin{tabular}{|c|c|c|c|c|c|c|}
\hline & \multirow[b]{2}{*}{$\mathbf{H}$} & \multirow[b]{2}{*}{0} & \multicolumn{4}{|c|}{ Semestre } \\
\hline & & & Fev/16 & Ago/16 & Fev/17 & Ago/17 \\
\hline \multicolumn{7}{|l|}{ Malvaceae } \\
\hline Sida cf rhombifolia & $\mathrm{T}$ & $\mathrm{N}$ & 1 & 1 & & \\
\hline \multicolumn{7}{|l|}{ Melastomataceae } \\
\hline Miconia sp & $\mathrm{T}$ & $\mathrm{N}$ & & & 1 & 1 \\
\hline Tibouchina granulosa (Desr.) Cogn. & $\mathrm{T}$ & $\mathrm{N}$ & 1 & 1 & 5 & 3 \\
\hline \multicolumn{7}{|l|}{ Myrtaceae } \\
\hline * Eugenia uniflora L. & $\mathrm{T}$ & $\mathrm{N}$ & & 1 & & \\
\hline \multicolumn{7}{|l|}{ Ochnaceae } \\
\hline Sauvagesia erecta L. & $\mathrm{O}$ & $\mathrm{N}$ & & 1 & 1 & 1 \\
\hline \multicolumn{7}{|l|}{ Phyllanthaceae } \\
\hline Phyllanthus tenellus Roxb. & $\mathrm{O}$ & $\mathrm{N}$ & 1 & 3 & & \\
\hline \multicolumn{7}{|l|}{ Plantaginaceae } \\
\hline Plantago major L. & $\mathrm{O}$ & $\mathrm{E}$ & 1 & & & \\
\hline \multicolumn{7}{|l|}{ Poaceae } \\
\hline Cynodum cf dactylum(L.) Pers. & $\mathrm{O}$ & $\mathrm{N}$ & & & & \\
\hline Eleusine indica (L.) Gaertn. & $\mathrm{O}$ & $E$ & & & & \\
\hline Melinis minutiflora P. Beauv. & $\mathrm{O}$ & $E$ & & & 1 & 1 \\
\hline Panicum maximum Jacq. & $\mathrm{O}$ & $\mathrm{N}$ & & 7 & & \\
\hline Paspalum sp & $\mathrm{O}$ & $\mathrm{N}$ & & & & \\
\hline ** Urochloa decumbens (Stapf) R.D.Webster & $\mathrm{O}$ & $\mathrm{E}$ & $79 \%$ & $87 \%$ & $67 \%$ & $62 \%$ \\
\hline \multicolumn{7}{|l|}{ Rubiaceae } \\
\hline Diodia sp & $\mathrm{O}$ & $\mathrm{N}$ & & 2 & & \\
\hline Richardia sp & $\mathrm{O}$ & $\mathrm{N}$ & 1 & & & \\
\hline \multicolumn{7}{|l|}{ Solanaceae } \\
\hline * Acnistus arborescens (L.) Schltdl. & $\mathrm{T}$ & $\mathrm{N}$ & & & 1 & \\
\hline ** Solanum americanum Mill. & $\mathrm{O}$ & $\mathrm{N}$ & 2 & 4 & & 1 \\
\hline Solanum granuloso-leprosum Dunal & $\mathrm{T}$ & $\mathrm{N}$ & & 1 & & \\
\hline outras gramíneas & & & $0 \%$ & $20 \%$ & $22 \%$ & $18 \%$ \\
\hline Total de plântulas & & & 52 & 121 & 60 & 49 \\
\hline Total de espécies & & & 30 & 36 & 21 & 20 \\
\hline
\end{tabular}

Fonte: Autores (2020)

Em que: $(F): T$ = árvore/arbusto e $\mathrm{O}$ = outras; e origem da espécie (O): $\mathrm{N}$ = nativa e $\mathrm{E}=$ exótica.

A evidente ocupação e recrutamento de regenerantes, após o plantio, foi constatada nos levantamentos de fevereiro e agosto de 2016 devido à crescente 
ocupação do espaço, tanto em porcentagem pelas gramíneas, especialmente pela invasora Urochloa decumbens, quanto por quantidade de plântulas e diversidade de espécies identificadas. Depois disso, nos levantamentos realizados em fevereiro e agosto de 2017, o número de plântulas e espécies registradas diminuiu, provavelmente devido à alta competição entre indivíduos e espécies presentes no local, bem como pelo sombreamento da área proporcionado pelo crescimento e cobertura de copa das mudas de espécies arbóreas plantadas no local. Assim, espécies colonizadoras iniciais, tal como a ocupação da Urochloa decumbens registrada no primeiro ano do plantio de mudas, podem inibir a ocupação por outras espécies devido à competição entre elas no local, retardando a substituição de espécies. Contudo, algumas espécies colonizadoras, tais como as arbóreas pioneiras, podem auxiliar na criação de condições que favoreçam a ocupação por outras espécies devido à eliminação de gramíneas invasoras, fato constatado em decorrência da intervenção antrópica por meio do plantio das mudas e o posterior crescimento destas após um ano. Essa matocompetição tem sido apontada como um dos principais problemas em plantios de restauração (RUIZ-JANSEN; AIDE, 2005; CHAVES et al., 2015; SANTOS et al., 2020).

Dentre as 58 espécies identificadas no período, 16 são arbóreas, das quais sete já haviam sido registradas no local (Actinistus arborescens, Erithryna crista-galli, Eugenia uniflora, Mimosa sp., Solanum americanum, Vernonanthura phosphorica e Vernonia sp.) e outras nove registadas como novas na área em processo de restauração florestal. Assim, resultados relacionados ao recrutamento de plântulas, especialmente em florestas tropicais, mostram que para que esse evento ocorra são necessárias boas condições locais para o estabelecimento de espécies arbóreas, bem como contínua dispersão de sementes da vegetação de áreas próximas na paisagem (SILVA et al., 2009; OLIVEIRA et al., 2015; CHAZDON; URIARTE, 2016). Então, a densidade do recrutamento de plântulas é reflexo do estado sucessional da floresta e apenas o plantio de árvores não garante que espécies lenhosas irão regenerar naturalmente a partir da paisagem circundante. Asteraceae foi a família de maior representatividade na amostragem. Isso 
ganha muita importância pelo fato desta representar cerca de $10 \%$ da flora mundial (BREMER, 1994). Outra família muito representativa na amostragem foi Fabaceae, uma das mais ricas em espécies nas florestas estacionais semidecíduas de domínio de Mata Atlântica (LEITÃO-FILHO, 1987; OLIVEIRA-FILHO; FONTES, 2000). Poaceae, também muito presente, habita principalmente locais abertos, como campos naturais e antrópicos, mas também sub-bosques, onde desempenham papel ecológico importante no revestimento do solo e ciclagem de nutrientes (SHIRASUNA et al., 2013).

Sendo assim, torna-se evidente a importância do monitoramento de longo prazo dentro e fora de áreas protegidas, não apenas devido aos processos antrópicos, mas também aos processos ecológicos naturais que podem influenciar as mudanças populacionais (STEM et al., 2005; SINCLAIR et al., 2007; WESTERN et al., 2009). Da mesma forma, plantios de restauração, prinipalmente com alta diversidade, podem atuar como catalisadores da sucessão secundária, incentivando o processo de regeneração natural (VIANI et al., 2010).

\section{CONCLUSÕES}

Apesar do número considerável de espécies amostradas na chuva de sementes, na sua grande maioria composta por indivíduos de hábito arbóreo, numericamente houve a presença maciça da espécie invasora Urochloa decumbens. No que diz respeito à regeneração natural, a participação das sementes de espécies arbóreas no total amostrado diminuiu comparativamente a chuva de sementes. Os registros observados evidenciam o início de um processo de regeneração natural, estimulado pelos próprios indivíduos plantados e pelo remanescente florestal presente nessa Unidade de Conservação. Contudo, apesar desse potencial, foi verificado que a Unidade de Conservação se encontra sob forte pressão, principalmente por parte das espécies herbáceas invasoras. Sendo assim, como forma de incentivo ao processo de regeneração, são necessárias medidas de manejo dessas espécies invasoras, para que os diásporos das espécies arbóreas nativas presentes tenham condições para se estabelecer e aumentar a diversidade local. 


\section{AGRADECIMENTOS}

Os autores agradecem à BR Consultoria Ambiental.

\section{REFERÊNCIAS}

BARBOSA, K. C.; PIZO, M. A. Seed Rain and Seed Limitation in a Planted Gallery Forest in Brazil. Restoration Ecology, Malden, v. 14, n. 4, p. 504-511, dec. 2006.

BARBOSA, L.M.; SHIRASUNA, R. T.; LIMA, F. C.; ORTIZ, P. R.; BARBOSA, K. C.; BARBOSA, T. C. Lista de espécies indicadas para restauração ecológica nas diferentes regiões do estado de São Paulo. São Paulo: Secretaria de Estado do Meio Ambiente, 2017. 344p.

BARROS, F.; MAMEDE, M. C. H.; MELO, M. M. R. F.; LOPES, E. A.; JUNG-MENDAÇOLLI, J.; MIRIZAWA, M.; MUNIZ, C. F. S.; MAKINO-WATANABE, H.; CHIEA, S. A. C.; MELHEM, T. S. A. A Flora filogenética do PEFI: composição, afinidades e conservação. In: BICUDO, D.C.; FORTI, M. C.; BICUDO, C.E.M. (org.). Parque Estadual das Fontes do Ipiranga (PEFI): unidade de conservação que resiste à urbanização de São Paulo. São Paulo: Secretaria do Meio Ambiente do Estado de São Paulo, 2002.p.93-110.

BATTILANI, J.L. Chuva de sementes em trecho de Floresta ripária, Mato Grosso do Sul, Brasil. 2010. 158f. Tese (Doutorado em Ecologia e Conservação) -Universidade Federal do Mato Grosso do Sul, Campo Grande, 2010.

BRANCALION, P. H. S.; GANDOLFI, S.; RODRIGUES, R. R. Restauração florestal. São Paulo, Brasil: Oficina de Textos, 2015. 432p.

BRANCALION, P.H.S.; RODRIGUES, R. R.; GANDOLFI, S.; KAGEYAMA, P. Y.; NAVE, A. M.; GANDARA, F. B.; BARBOSA, L. M.; TABARELLI, M. Instrumentos legais podem contribuir para a restauração de florestas tropicais biodiversas. Revista Árvore, Viçosa, v. 34, n. 3, p.455-470, set. 2010.

BRASIL. Lei n. 9.985, de 18 de junho de 2000. Disponível em: http://www.planalto.gov.br/ ccivil_03/leis/L9985.htm. Acesso em: 14 abril 2015.

BREMER, K. Asteraceae: cladistics and classification. Portland: Timber Press, 1994. 752p.

CHAVES, R.B.; DURIGAN, G.; BRANCALION, P. H. S.; ARONSON, J. On the need of legal frameworks for assessing restoration projects success: new perspectives from São Paulo state (Brazil). Restoration Ecology, Malden, v. 23, n. 6, p. 754-759, sep. 2015.

CHAZDON, R.L.; URIARTE, M. Natural regeneration in the context of large-scale forest and landscape restoration in the tropics. Biotropica, Nova Jersey, v. 48, n. 6, p. 709-715, 2016.

CÔRTES, M.C.; URIARTE, M. Integrating frugivory and animal movement: a review of the evidence and implications for scaling seed dispersal. Biological Reviews, Cambridge, v. 88, p. 255-272, nov. 2013. 
ENGEL, V. L.; PARROTTA, J.A. Definindo a restauração ecológica: tendências e perspectivas mundiais. In: KAGEYAMA, P. Y.; OLIVEIRA, R. E.; MORAES, L. F. D.; ENGEL, V. L.; MENDES, F. B. G. (org.). Restauração de ecossistemas naturais. Botucatu: FEPAF, 2003. p. 3-26.

FERREIRA, D.F. Sisvar: a computer statistical analysis system. Ciência e Agrotecnologia, Lavras, v. 35, n. 6, p. 1039-1042, 2011.

FONSECA, D.A.; BACKES, A. R.; ROSENFIELD, M. F.; OVERBECK, G, E.; MULLER, S. C. Avaliação da regeneração natural em área de restauração ecológica e mata ciliar de referência. Ciência Florestal, Santa Maria, v. 27, n. 2, p. 521-534, jun. 2017.

GELDMANN, J.; BARNES, M; COAD, L.; CRAIGIE, I. D.; HOCKINGS, M.; BURGESS, N. D. EffectivenesS of terrestrial protected areas in reducing habitat loss and population declines. Biological Conservation, Washington, v. 161, p. 230-238, may 2013.

GROMBONE-GUARATINI, M. T.; RODRIGUES,R. R. Seed bank and seed rain in a seasonal semideciduous forest in South-eastern Brazil. Journal of Ecology, Londres, v. 18, n. 1, p. 759774 , sep.2002.

HOOPER, E.; LEGENDRE, P.; CONDIT R. Barries to forest regeneration of deforested and abandoned land in Panama. Journal of Applied Ecology,v. 42, p. 1165-1174, 2005.

HOWE, H. F.; SMALLWOOD, J. Ecology seed dispersal. Annual Review of Ecology, Evolution and Systematics, Palo Alto, v. 13, p. 201-228, 1982.

LAMB, D.; ERSKINE, P. D.; PARROTTA, J.A. Restoration of Degraded Tropical Forest Landscapes. Science, Washington, v. 310, p. 1628-1632, dec. 2005.

LEITÃO-FILHO, H.F. Considerações na florística das florestas tropicais e subtropicais no Brasil. Revista do Instituto de Pesquisas Florestais, Piracicaba, v. 35, p. 41-46, 1987.

LEITE, M.S.; TAMBOSI, L. R.; ROMITELLI, I; METZGER, J. P. Landscape Ecology Perspective in Restoration Projects for Biodiversity Conservation: a Review. Brazilian Journal of Nature Conservation, Rio de Janeiro, v. 11, n. 2, p. 108-118, dec.2013.

MELO, F. P. L.; DIRZO, R.; TABARELLI, M. Biased seed rain in forest edges: evidence from Brazilian Atlantic forest. Biological Conservation, Washington, v. 132, p. 50-60, sep. 2006.

OLIVEIRA, M.A.M.; OLIVEIRA, A. C.; ROSSI, L.; CATHARINO, E. L. M.; GOMES, E. P. C.; SANTOS JUNIOR, N. A. Dinâmica da regeneração natural em uma floresta baixa de restinga degradada. Hoehnea, São Paulo, v. 42, n. 4, p. 759-774, dez. 2015.

OLIVEIRA-FILHO, A. T.; FONTES, M.A. Patterns of floristic differentiation among Atlantic Forests in southeastern Brazil and the influence of climate. Biotropica, Nova Jersey, v. 3, p. 793-810, mar. 2000.

RODRIGUES, A. S. L.; AKÇAKAYA, H. R.; ANELMAN, S. J.; BAKARR, M. I.; BOITANI, L.; BROOKS, T. M.; CHANSON, J. S.; FISHPOOL, L. D. C.; FONSECA, G. A. B.; GASTON, K. J.; HOFFMANN, M.; MARQUET, P. A.; PILGRIM, J. D.; PRESSEY, R. L.; SCHIPPER, J.; SECHREST, W.; STUART, S. N.; UNDERHILL, L. G.; WALLER, R. W.; WATTS, M. E. J.; YAN, X. Global gap analysis: priority regions for expanding the global protected-area network. Bioscience, Oxford, v. 54, p. 1092-1100, dez. 2004. 
RODRIGUES, R.R.; GANDOLFI, S.; NAVE, A. M.; ARONSON, J.; BARRETO, T. E.; VIDAL, C. Y.; BRANCALION, P. H. S. Large-scale ecological restoration of high-diversity tropical forests in SE Brazil. Forest Ecology and Management, Amsterdan, v. 261, p. 1605-1613, may 2011.

RUIZ-JANSEN, M. C.; AIDE, M. Restoration Success: How is it being measured? Restoration Ecology, Malden, v. 13, n. 3, p. 569-577, sep. 2005.

SANTOS, F.A.M.; LELES, P. S. S.; RESENDE, A. S.; NASCIMENTO, D. F.; SANTOS, G. R. Estratégias de controle de braquiárias Urochloa spp. na formação de povoamento para restauração florestal. Ciência Florestal, Santa Maria, v. 30, n. 1, p. 29-42, abr. 2020.

SANTOS, P.M.; FUNARI, F. L. Clima local. In: BICUDO, D.C.; FORTI, M.C.; BICUDO, C.E.M. (org.). Parque Estadual das Fontes do Ipiranga (PEFI):unidade de conservação que resiste à urbanização de São Paulo. São Paulo: Secretaria do Meio Ambiente do Estado de São Paulo, 2002, p. 29-48.

SÃO PAULO. Plano de Manejo do Parque Estadual das Fontes do Ipiranga: resumo executivo. São Paulo: SMA, 2008. 34p.

SHIRASUNA, R. T.; FILGUEIRAS, T. S.; BARBOSA L. M. Poaceae do Rodoanel Mario Covas, Trecho Sul, São Paulo, SP, Brasil: florística e potencial de uso na restauração de áreas degradadas. Hoehnea, São Paulo, v. 40, n. 3, p. 521-536, set. 2013.

SILVA, B. G.; KOCH, I.; SILVA, W.R. Fruit production along footpaths in an Atlantic rain forest area. Plant Ecology \& Diversity, Londres, v. 11, n. 1, p. 41-53, abr. 2018.

SILVA, C.R.; BARBOSA, J. M.; CARRASCO, P. G.; CASTANHEIRA, S. A.; PEREIRA, M. A.; SANTOS JUNIOR, N. A. Chuva de sementes em uma floresta alta de restinga em Ilha Comprida (SP). Cerne, Lavras, v. 15, p. 355-365, set. 2009.

SILVA, F.R.; MONTOYA, D.; FURTADO, R.; MEMMOTT, J.; PIZO, M. A.; RODRIGUES, R. R. The restoration of tropical seed dispersal networks. Restoration Ecology, Malden, v. 23, n. 6, p. 852-860, jul.2015.

SINCLAIR, A.R.E.; MDUMA, S.A.R.; HOPCRAFT, J.G.C; FLYXELL, J.M.; HILBORN, R.; THIRGOOD, $S$. Long-term ecosystem dynamics in the Serengeti: Lessons for conservation. Conservation Biology, Washington, v. 21, p. 580-590, may 2007.

STEM, C.; MARGOLUIS, R.; SALAFSKY, F.; BROWN, M. Monitoring and evaluation in conservation: a review of trends and approaches. Conservation Biology, Washington, v. 19, p. 295-309, nov.2005.

WESTERN, D.; RUSSELL, S.; CUTHILL, I. The status of wildlife in protected areas compared to non-protected areas of Kenya. PLoS One, São Francisco, v. 4, n. 7, p. 1-6, fev. 2009.

VAN DER PIJL, L. Principles of seed dispersal in higher plants. Berlim: Springer-Velag, 1982. $215 p$.

VIANI, R. A. G.; DURIGAN, G.; MELO, A. C. G. A regeneração natural sob plantações florestais: desertos verdes ou redutos de biodiversidade? Ciência Florestal, Santa Maria, v. 20, n. 3, p. 533-552, set. 2010. 


\section{Contribuição de Autoria}

\section{1 - Karina Cavalheiro Barbosa}

Bióloga, Dra., Pesquisadora Autônoma

https://orcid.org/0000-0002-0856-1510•cbkarina@yahoo.com

Contribuição: Conceituação, Curadoria de dados, Análise Formal, Investigação, Visualização de dados, Escrita - primeira redação

\section{2 - Eduardo Luis Martins Catharino}

Engenheiro Agrônomo, Dr., Pesquisador Científico

https://orcid.org/0000-0002-0990-8758•mcatarin@uol.com.br

Contribuição: Análise Formal, Investigação, Metodologia, Supervisão, Escrita - primeira redação

\section{3 - Luiz Mauro Barbosa}

Engenheiro Agrônomo, Dr., Pesquisador Científico

https://orcid.org/0000-0001-9874-059X•Imbarbosa@ibot.sp.gov.br

Contribuição: Obtenção de financiamento, Investigação, Administração do projeto

\section{4 - Hilton Thadeu Zarate do Couto}

Engenheiro Agrônomo, Dr., Professor

https://orcid.org/0000-0002-8698-6442•htzcouto@usp.br

Contribuição: Análise Formal, Investigação, Metodologia, Visualização de dados

\section{5 - Nelson Augusto dos Santos Junior}

Biólogo, Dr., Pesquisador Científico

https://orcid.org/0000-0002-9601-2350•njunior@ibot.sp.gov.br

Contribuição: Conceituação, Análise Formal, Investigação, Metodologia, Supervisão, Visualização de dados e Escrita - revisão e edição

\section{Como citar este artigo}

Barbosa, K. C.; Catharino, E. L. M.; Barbosa, L. M.; Couto, H. T. Z.; Santos Junior, N. A. Potencial de regeneração natural de um plantio compensatório realizado em unidade de conservação urbana sob forte pressão antrópica. Ciência Florestal, Santa Maria, v. 31, n. 2, p. 786-807, 2021. DOI 10.5902/1980509843659. Disponível em: https://doi.org/10.5902/1980509843659. Acesso em: xx mês-abreviado 2021. 